\title{
Fabrication and Vibration Results of 30-cm Pyrolytic Graphite Ion Optics
}

\author{
Michael K. De Pano ${ }^{*}$, Stephen L. Hart ${ }^{\dagger}$, Andrew A. Hanna ${ }^{\ddagger}$, and Analyn C. Schneider ${ }^{\S}$ \\ Boeing Electron Dynamic Devices, Inc., Torrance, California, 90509
}

\begin{abstract}
Boeing Electron Dynamic Devices, Inc. is currently developing pyrolytic graphite (PG) grids designed to operate on 30-cm NSTAR-type thrusters for the Carbon Based Ion Optics (CBIO) program. The PG technology effort of the CBIO program aims to research PG as a flightworthy material for use in dished ion optics by designing, fabricating, and performance testing $30-\mathrm{cm}$ PG grids. As such, PG grid fabrication results will be discussed as will PG design considerations and how they must differ from the NSTAR molybdenum grid design. Surface characteristics and surface processing of PG will be explored relative to effects on voltage breakdown. Part of the CBIO program objectives is to understand the erosion of PG due to Xenon ion bombardment. Discussion of $P G$ and $C C$ sputter yields will be presented relative to molybdenum. These sputter yields will be utilized in the life modeling of carbon-based grids. Finally, vibration results of 30-cm PG grids will be presented and compared to a first-order model generated at Boeing EDD. Performance testing results of the PG grids will not be discussed in this paper as it has yet to be completed.
\end{abstract}

\section{Introduction}

B oeing Electron Dynamic Devices, Inc. (EDD) has recently developed 30-cm pyrolytic graphite grids for NASA Marshall Space Flight Center (MSFC). Aptly named, the Carbon Based Ion Optics (CBIO) program was originally a 16-month development effort funded by In-Space Propulsion Technology Project and NASA MSFC's Next Generation Electric Propulsion office. There are two phases to the CBIO program. The Phase 1 goals are to design, manufacture, and test 30-cm pyrolytic graphite and carbon-carbon grids. The grid development occurred in two parallel paths with two competing technologies being pursued to mitigate risk in the use of one carbon-based material over the other. Boeing EDD is leading the Pyrolytic Graphite (PG) grid development effort building upon previous $8-\mathrm{cm}$ and $30-\mathrm{cm}$ PG work done at NASA Glenn Research Center (GRC) ${ }^{1,2}$ while concurrently, the Jet Propulsion Laboratory (JPL) CBIO team is developing the Carbon-Carbon (CC) grid technology, ${ }^{3,4}$. Each CBIO team works independently, and collaborates fully to foster discussion and facilitate technology transfer.

The original program outline was organized such that each optics design would go through a performance test, and then a vibration test at NSTAR DS1 levels in order to determine the ability of the material and optics design to survive flight-like environmental conditions. Following vibration testing, each respective grid technology would then be subject to performance testing again to understand the effects of vibration. The performance requirements include increased design margin over the State of the Art (SOA) NSTAR molybdenum grids. At the conclusion of the test and vibration phase, a downselect of one grid technology was to occur with the winning carbon design moving forward to perform a $2 \mathrm{kHr}$ wear test at JPL designed to gather erosion. The information gathered from the wear test, sputter erosion measurements, and previous CBIO gridlet testing done by Colorado State University

\footnotetext{
- Program Manager, Electric Propulsion Organization, Member AIAA.

Member of Technical Staff, Electric Propulsion Organization, Non-member.

${ }^{\ddagger}$ Member of Technical Staff, Electric Propulsion Organization, Member AIAA.

$\S$ Member of Technical Staff, Electric Propulsion Organization, Non-member.
} 
$(\mathrm{CSU})^{5}$, will help validate the life modeling software currently being developed at JPL for the CBIO program. As wear testing winds down, both a final report and a Phase 2 proposal will be generated to ultimately develop the winning grid technology into larger 40 -cm ion optics.

Boeing EDD completed a pyrolytic graphite grid design in December of 2002 and presented it for critique and comment at the CBIO Preliminary Design Review (PDR) in February of 2003. The design was based on the NSTAR heritage molybdenum grids with accommodations made for pyrolytic graphite material properties and manufacturing considerations. Changes to the original screen grid design were necessary due to high mechanical stresses from an unforeseen consequence of the machining process This redesign effectively mitigated the problems seen on the earlier screen grids. Details of the design are presented in Section II, Paragraph D of this report.

A first order analysis of the new pyrolytic graphite

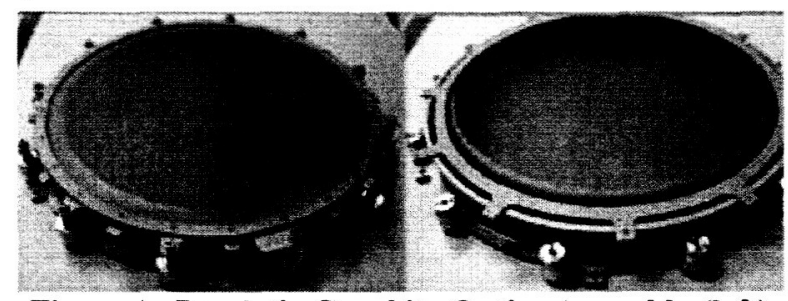

Figure 1. Pyrolytic Graphite Optics Assembly (left) and Assembly with Accel Grid Removed (right) optics assembly was completed by the $\mathrm{f}^{\mathrm{t}}$ Quarter Review, which also served as the Preliminary Design Review. This model served as a preliminary check for material behavior when subject to the given frequency spectrum. From this model, resonant frequencies and maximum deflection amplitudes were determined for each grid and compared to the actual gap. The results of this analysis indicate that the maximum deflection of each of the grids would not bridge the inter-grid gap. These results were presented at the PDR for critique and comment. The major action from the PDR was to validate the model with molybdenum grids, which are known to deflect in large enough amplitudes to bridge the gap. The model was then updated to accommodate molybdenum grids. The results of the first order analysis show that the deflection amplitude of the molybdenum grids are within $9 \%$ of actuals .

The model also predicts the natural frequencies of the PG and molybdenum grids. The predicted value of the molybdenum accel grid natural frequency versus the actual value was shown to be $8 \%$. The actual value of the molybdenum screen grid natural frequency was not measured. Therefore, the accuracy of the first order predictions to within $9 \%$ for molybdenum grid displacements and $8 \%$ for predicted molybdenum accel grid natural frequency provided confidence that the PG grid model was a within reason. The PG grid model predicted that the vibration test would not result in grid contact at NSTAR Acceptance Levels.

The PG optics assembly, PG3, completed vibration testing on February 27, 2004 to NSTAR Acceptance Levels (9.1 Grms). The optics assembly was mounted to NSTAR engineering model EMT-1C and tested at EDD's environmental facilities in Torrance, CA. As predicted, there was no grid to grid contact. This was verified by a simple open circuit using grid contact as a switch. An oscilloscope was set to trigger if there was grid-to-grid contact. The predicted natural frequency of the accel grid showed an error from the model of $13 \%$, which was expected for a first order characterization. Post-vibration inspection did not reveal any damage to either the accel or the screen grids. Post-vibration changes in concentricities and gaps were negligible. The results of this vibration test will be discussed in Section III.

\section{Pyrolytic Graphite Grid Design}

\section{A. Pyrolytic Graphite Material Basics}

Pyrolytic graphite is formed through a pyrolysis reaction. This process consists of decomposing a purified hydrocarbon gas, usually methane, at high temperature in a vacuum. The freed carbon atoms then deposit on a mandrel taking on its shape. Assuming the mandrel was flat on a horizontal plane, the first layer of carbon atoms forming on the mandrel and parallel to its surface is called the A-plane. The atomic bonds taking place in this plane use a hybrid sp2-triagonal orbital, or sigma-bond, which is the basis for all graphitic structures ${ }^{6}$. The very next atomic layer of carbon, called the B-plane, will also form using the in-plane sigma bond; however, the atoms of carbon are horizontally offset from the atoms in the A-plane. The next layer (back to an A-plane) will then offset from the B-plane structure such that the atoms of carbon line up directly over the first A-plane. Therefore, the stacking sequence of atoms occurs in an A-B-A-B-A-... manner thickening the overall graphitic structure as more layers are deposited. The direction perpendicular to the A-B plane is known as the Gdirection, or "growth" direction. The resulting lattice structure is therefore highly anisotropic in the C-direction ${ }^{6}$. Atomic bonds in the horizontally planar A-B layers, known as sigma bonds, are strong due to the short distances between neighboring atoms and valence states of electrons. However, atomic bonds are much weaker in the perpendicular inter-layer Cdirection ${ }^{6}$ where pi bonding takes place and neighboring atomic spacing is greater

$$
2
$$

American Institute of Aeronautics and Astronautics 
Because of these anisotropic properties, thermal conductivity is relatively high in the A-B planes, but very low, in the $C$ direction. $P G$ is has good thermal conduction in the A-B planes, but at best is an average thermal insulator in the $\mathrm{C}$-direction ${ }^{6}$. Electrical resistivity due to the anisotropic structure shows conductor-like resistances in the A-B plane, and almost that of a weak insulator in the $\mathrm{C}$ plane. Other characteristics affected by this anisotopy are thermal expansion characteristics, mechanical strength, and magnetic properties. Unfortunately, the weaker pibonding structures make pyrolytic graphite susceptible to delamination if shearing stesses are present.

\section{B. Comparison to State - f-the-Art NSTAR}

For the CBIO program, an optical design tailored to carbon grids was provided to Boeing EDD by JPL. Design parameters provided were aperture diameters for both the screen and accel grids, center-to-center aperture spacing, grid spacing, dish depth, and associated voltages. Boeing EDD was tasked to provide a flight-like mechanical layout of the optics assembly that complied with the electrical parameters. Boeing EDD chose to utilize a modified

\begin{tabular}{lcc}
\hline \multicolumn{1}{c}{ Parameter } & NSTAR & CBIO \\
\hline Grid Material & Moly & PG \\
Screen Aperture Diameter & 1.0 & .97 \\
Screen Grid Thickness & 1.0 & 1.3 \\
Accel Aperture Diameter & 1.0 & 1.0 \\
Accel Grid Thickness & 1.0 & 1.4 \\
Spacing & 1.0 & 1.3 \\
Center-center spacing & 1.0 & 1.0 \\
\hline
\end{tabular}

Table 1. Comparison of NSTAR and CBIO Parameters
NSTAR optics assembly design tailored for carbon grid material. The NSTAR optics assembly design utilized dished molybdenum grids which each used rivets to attach to their respective molybdenum grid stiffener at the outer flange portion of the grids. The screen grid is mounted to its grid stiffener and then mounted to the optics mounting ring with fasteners. The accel grid is mounted to its respective molybdenum grid stiffener and then mounted to the optics isolator stack, which were attached to the optics mounting ring. The accel grid was shimmed accordingly to achieve the desired grid gap. The flight grids received selective surface preparation to facilitate the adherence of sputtered grid material.

\section{Pyrolytic Graphite Grid Fabrication}

The pyrolitic graphite used in the CBIO program consists of a proprietary process for fabricating PG with free standing structures in mind, rather than the more typical application, such as those used in coatings. This process results in PG that is also better for machining and is more resistant to delamination. The surface finish of the

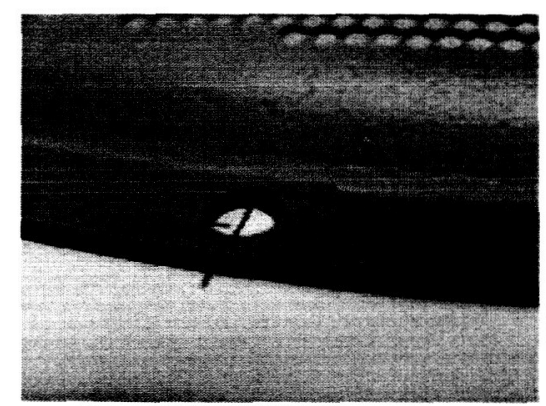

Figure 2. Delamination on screen grid

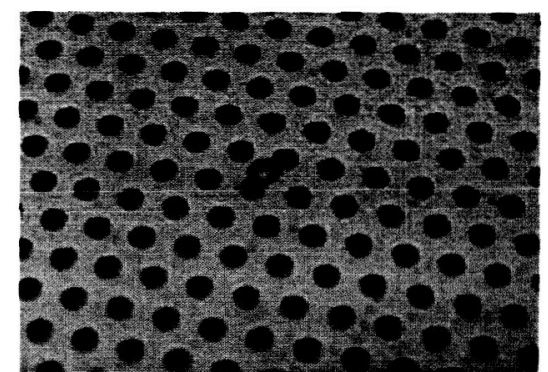

Figure 3. Chip-out on accel grid surface mandrel on which the pyrolytic graphite structure is grown will, in part, help determine the atomic structure of the grown layers. Surface defects in the mandrel due to machining or dust particles will be a site of growth for columnar-like structures within the material. As the thickness of the pyrolytic graphite increases, the columnar grain structure increases in size. Because large columnar grain structures are not desirable, new growth sites are introduced by addition of impurities during the growth phase. The addition of new growth sites produces columnar like structures that do not originate at the mandrel, but in the bulk of the graphite thus interrupting any large columnar growths ${ }^{6}$.

The PG grids are grown on shaped mandrels where they take on the desired geometry. Once they are machined to the correct thickness, they are heat treated higher than the deposition temperature to relieve stresses induced by the machining ${ }^{3}$. The result is a grid blank that is of the desired shape and thickness, and is now ready for the apertures.

\section{Grid Procurement and Assembly Results}

Initially, 2 sets of $P G$ grids were ordered, plus 1 set of grid blanks for risk mitigation. The first gridset (PG1) processing differed from the second gridset (PG2) in that PG1 did not have a heat treat performed after the machine to thickness step. Results of the completed gridsets were mixed. Data was taken at both the

3

American Institute of Aeronautics and Astronautics 
grid machining house after final thickness machine, but before the apertures were drilled, and then independently verified by a Boeing EDD vendor after delivery.

PG1 did not meet the spherical radius specifications on either the screen or accel grids. Overall, the surface characteristics of the grids showed the typical PG "dimpled" surface. Minor "chip -outs" were present, which were generally more pronounced on the accel grid since the hole diameters are smaller thus having more surface to show any chip-outs. The grids were assembled into the optics assembly to understand their concentricity and spacing characteristics. As expected, the out of tolerance spherical radius affected the grid gap spacing such that the design gap could not be maintained over the full diameter of the grids. After all measurements were made, a cursory inspection at high magnification revealed cracks on a downstream section of the outer row of screen grid apertures. It was indeterminate if the cracks existed before the assembly process began, as inspection at high magnification was not done upon delivery of the grids.

PG2 also did not meet spherical radius specifications, however, the discrepancy was less than PG1. A few areas of the accel grid showed chip-outs that were quite deep, some on the order of an accel hole diameter (Figure 3). However, they did not protrude through the accel grid. The screen grid showed some small delaminations at the periphery which were later determined to be the result of an incomplete clean-up machining operation (Figure 2). Because these defects brought to light how machining operations were performed for this design, valuable lessons were learned for machining the next gridset. PG2 followed the same assembly process as PG1 with the exception that previous to assembly, a high magnification inspection was done on both the screen and accel grids. No cracks were found on either grid. However, after assembly a high magnification inspection revealed cracking along a section of the outer rows of the downstream side of the screen grid (Figure 4). Because this was the second screen grid with similar cracks, an investigation was initiated to determine root cause.

Results of the investigation showed that the flange portions of each of the screen grids had a slight "cant" in the downstream direction as one moves radially outward. The cant also existed in a similar range for the PG blanks indicating that this condition was in some way the result of the machining process. The cant revealed a weak point in the screen grid design that may have surfaced as a problem during the vibration phase of the program.

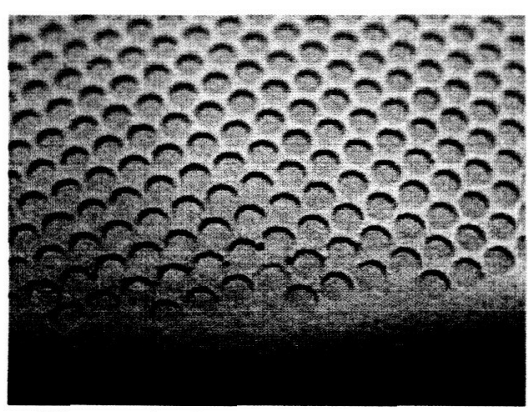

Figure 4. Cracks on screen grid Mechanical analysis carried out by Boeing EDD engineers recommended a solution that designed the assembly to distribute stress more evenly across the grid. After appropriate measures were taken to eliminate the root cause of the screen grid cracks, PG3 fabrication was started. Inspection of the completed gridsets shows they were within print tolerances. The surface characteristics of both screen and accel grids were better than those of PG1 and PG2. No delaminations were seen, and chip-outs were of minimal depth and few in number. Assembly of the grids indicated equivalent concentricities as both PG1 and PG2, and additionally, the uniformity of spacing well exceeded that of PG1 and PG2 (Figure 5). Inspection at magnification after assembly into the stiffening rings showed no signs of screen grid cracks. 


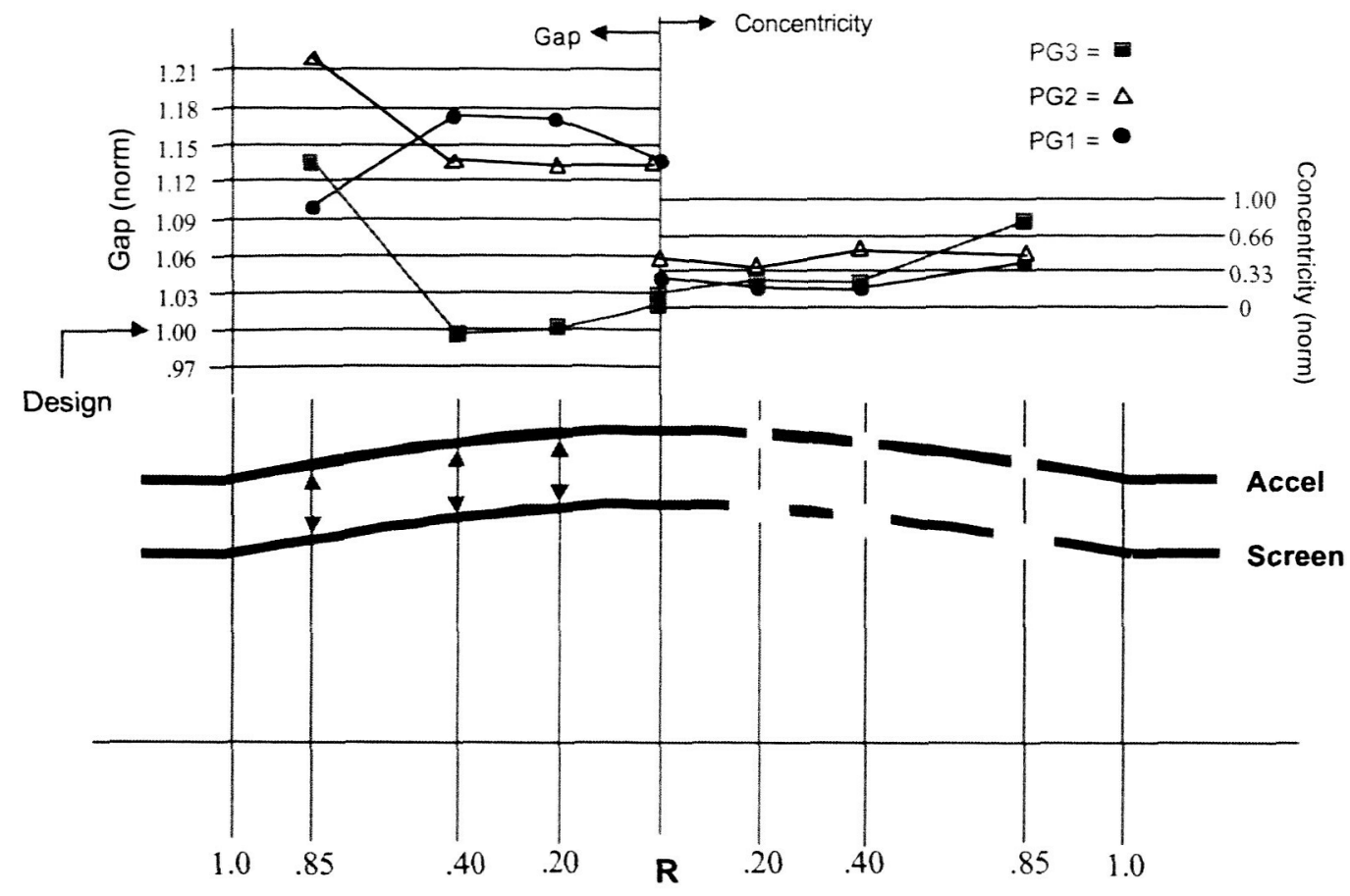

Figure 5. Summary of Pyrolytic Graphite Grid Assembly Data

\section{Vibration Testing Results}

\section{E. Test Results}

Although the original program plan was to perform a baseline performance measurement previous to the vibration testing, program constraints changed this plan. Previous success of vibration testing at Boeing EDD's facility with the program's carbon-composite gridset ${ }^{4}$ provided a good pathfinder for vibrating the PG3 gridset. PG3's accel grid was instrumented with two, 5-gram accelerometers at downstream positions identified by Boeing EDD's mechanical analysts, and one accelerometer on the outer edge of the grid. The screen grid was instrumented with two accelerometers on the upstream side. However, in order to prevent damage to the thin screen grid webbing, both accelerometers were positioned on the outer edge of the grid, which ultimately did not provide sufficient data for determination of the screen grid natural frequency. The grid assembly was then installed on

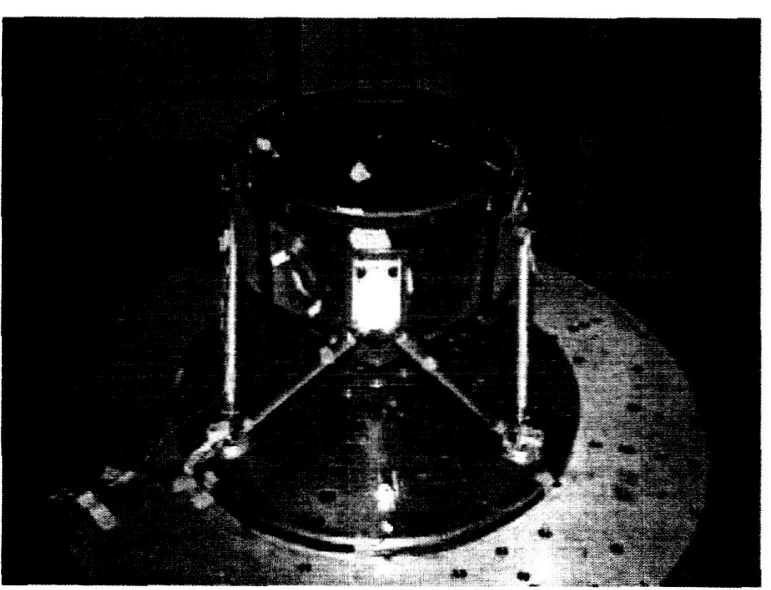

Figure 6. PG optics assembly vibration setup on EMT-1 C thruster EMT-1C provided by NASA GRC (Figure 6), which is the same thruster used in the NSTAR protoflight vibration testing. An accelerometer was also installed on the thruster mounting ring to understand the actual input spectrum to the optics assembly. A grid contacting circuit was instrumented between the accel and screen grids to monitor any grid-to-grid contact with a triggering oscilloscope. The contact circuit setup was tested before each vibe to insure that grid contact, if it occurred, would be seen. An initial sine sweep was performed to understand the frequency characteristics of the assembly The thruster was then vibration tested to NSTAR acceptance random vibration levels of 9.1 grms for 1 minute. The vibration test, including ramp up to full-level and then down, had a total duration of 3 minutes. There was no gridto-grid contact or damage observed. Comparison of the pre-and post-test low-level response of each grid showed no hidden damage. Post-test grid gap and concentricity measurements were essentially unchanged, and visual inspection under magnification did not reveal any damage or delaminations from the vibration test. 
F. Comparison to Model

A first-order mechanical analysis of the CBIO PG grids was performed at EDD to evaluate the natural frequencies and deflections of the grids under the NSTAR launch conditions and to determine if the PG design provides adequate clearance between the two grids to

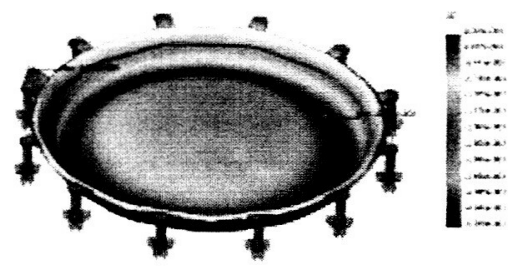

ACCEL GRID

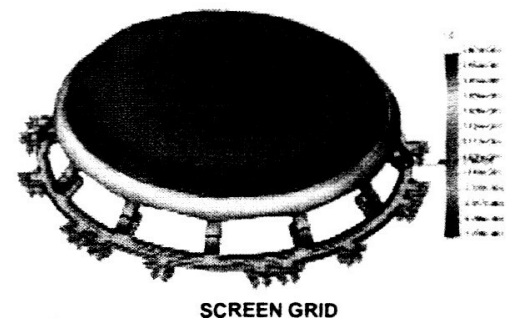

SCREEN GRID prevent contact. Using micro

Figure7. PG Grid Model Results

models to calibrate material characteristics, finite element models of the grids and their support structures were used to analyze the responses and deflections of each grid (Figure 7). The combined deflection of the two grids showed the PG design is adequate for avoiding grid contact.

In order to validate the PG analysis, the same first-order analysis was applied to modeled NSTAR molybdenum grids. The model did verify that the clearance provided by the molybdenum design was not adequate for avoiding grid contact. This was consistent with the grid contact observed during previous vibration testing of the molybdenum grids.

Actual resonant frequencies of the PG3 accel grid measured during testing compared well with those predicted from the first-order model. The fidelity of the model was improved by using the results from NSTAR DS1's vibration data and calibrating the model ${ }^{7}$. The model was able to predict the resonant frequency of the PG accel grid to with $13 \%$ (Table 2 ). These are excellent results for a first order model.

\begin{tabular}{lcccccc}
\hline & $\begin{array}{c}\text { Moly-NSTAR } \\
\text { NASA } \\
\text { (analysis) }\end{array}$ & $\begin{array}{c}\text { Moly-NSTAR } \\
\text { NASA }^{7} \\
\text { (test) }\end{array}$ & $\begin{array}{c}\text { Moly-NSTAR } \\
\text { EDD } \\
\text { (analysis) }\end{array}$ & $\begin{array}{c}\text { PG-NSTAR } \\
\text { GRC } \\
\text { (test) }\end{array}$ & $\begin{array}{c}\text { PG-CBIO } \\
\text { EDD } \\
\text { (analysis) }\end{array}$ & $\begin{array}{c}\text { PG-CBIO } \\
\text { EDD } \\
\text { (test) }\end{array}$ \\
\hline Accel natural frequency & 1.08 & $1.0(\mathrm{NOM})$ & 1.08 & $1.0(\mathrm{NOM})$ & 1.0 & 0.87 \\
\hline
\end{tabular}

Table 2. Comparison of Model to Measured Grid Natural Frequencies

\section{Surface Testing Results}

\section{G. Voltage Breakdown Results}

To understand voltage holdoff of pyrolytic graphite and carbon composite materials, JPL and Boeing EDD performed breakdown testing utilizing PG

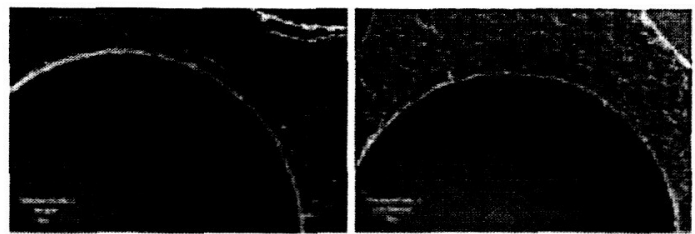

Figure 9. Untreated (left) and treated (right) samples of varying surface conditions. The vacuum setup utilized a graphite ball held at ground potential relative to the carbon sample, which was biased negatively with respect to

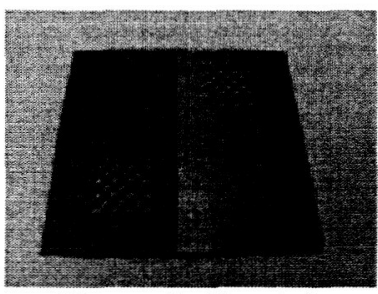

Figure 8. Treated Surface (Left-Hand Side)

voltage was measured as a function of separation distance between the ball and the sample. Discussion of the process and setup is described in further detail in Reference [3]. Results of the pyrolytic graphite testing is summarized in this paper, but full data sets and conclusions will be published at a later date by JPL. For this testing, Boeing EDD provided 2"x2" squares of pyrolytic graphite with varying surface conditions. 
The samples had surface finishes in the finished grown state without any machining. An interesting artifact of the growing process is seen on each side of the sample. The mandrel side will have "dimples" that are a result of the surface finish of the mandrel. Much like a golf ball, the dimples protrude into the surface of the sample, or are concave. The growth side of the sample shows convex surface features. This is consistent with how PG is grown and what is typically seen on both the upstream and downstream surfaces of the PG grids. Apertures were then drilled into the PG material and similarly tested to determine the voltage holdoff effects. The results showed a net decrease in voltage holdoff ability and also showed that there was essentially no difference between concave or convex sides as the edge effects of the apertures dominated. Of most interest however, is the increase in voltage holdoff due to a proprietary treated surface.

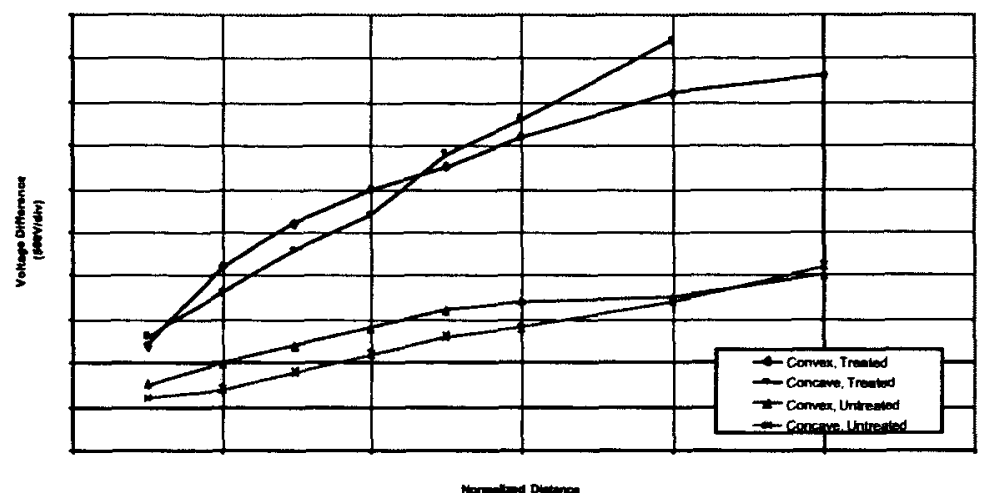

Figure 10. Gritblast Effects on Voltage Breakdown machined with a finish equivalent to the as-received pyrolytic graphite grids (Figure 8). One-half of the coupon was then treated to achieve a surface finish as seen in Figure 9. Results indicate that the effect of gritblasting has dramatically improved the voltage holdoff capability of the material as seen in Figure 10. The surface appears to be more uniform with burrs removed and rounded edges. It is also evident that neither convex nor concave dimples play a major role in voltage breakdown once the surface is treated.

\section{H. Sputter Yield Results}

Of large interest for programs utilizing carbon based grids are the sputter yields of both pyrolytic graphite and carbon-carbon composite materials used on the CBIO program. Colorado State University was tasked with

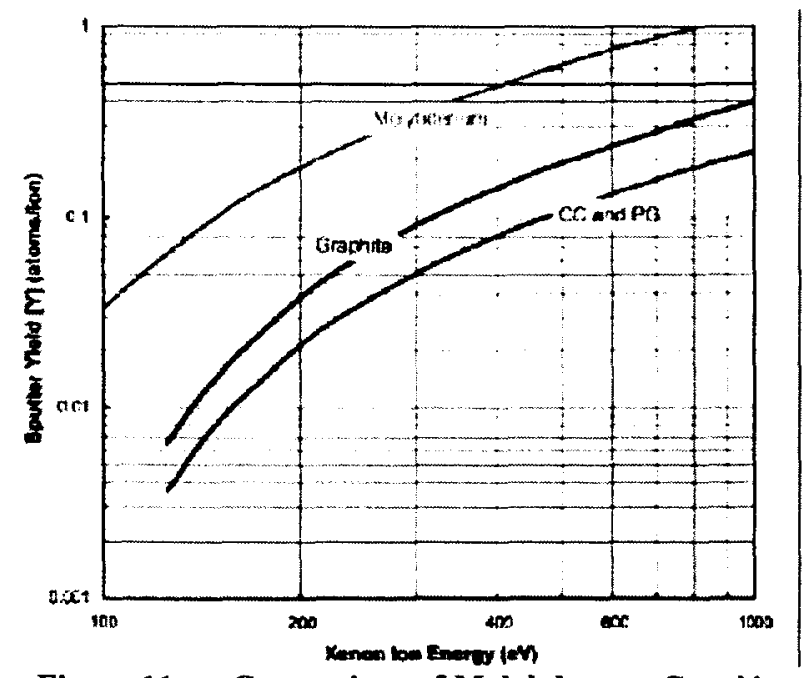

Figure 11. Comparison of Molybdenum, Graphite, CC, and PG Total Sputter Yields at Normal Incidence ${ }^{8}$ determining these yields on the subject materia ls by bombarding the carbon sample surfaces with Xenon ions at various ion energies and incidence angles. These measurements were done by directing a Xenon ion beam onto the carbon sample under test. The target was able to be rotated through angles ranging from 0 to $60^{\circ}$ relative to the incident ion beam. A quartz crystal microbalance (QCM) recorded the sputtered carbon distribution above the target, which was used to determine differential sputter yields and total sputter yields. While the complete task and results of CSU's work are presented in Ref [8], a summary comparing total sputter yields at normal incidence for CC, PG, molybdenum, and POCO graphite is shown in Figure 11. Characterization of the differential and total sputter yields by CSU has resulted in confirmation of the expected lifetimes that can be achieved if these carbon-based materials were utilized on gridded ion thrusters. CSU test results indicate that PG and $\mathrm{CC}$ sputter yields are virtually identical. Furthermore, relative to equivalent molybdenum grids, the expected lifetimes of PG and CC grids would be 7 - 9 times longer for Xenon ion bombardment energies 
ranging from $300-1000 \mathrm{eV}$, respectively. For energies below $300 \mathrm{eV}$, it is expected that there could be an even more significant increase in propellant throughput (and life time) $)^{3}$.

\section{Conclusion}

The CBIO program encompasses developing both the PG and CC technologies such that the benefits of each of the materials can quantified. Questions regarding survival of vibration, grid manufacturability, voltage holdoff, and sputter erosion are goals that the CBIO program aims to answer. The importance of the data acquired by the CBIO program is helping current and future programs make system level trades based on a better understanding of feasibility and expected lifetimes of carbon based grids. The quality of PG grids has improved as both Boeing EDD and its PG grid vendor work together to overcome the issues involving the fabrication and machining of PG. The result is a $30-\mathrm{cm}$ design that is a manufacturable, able to be assembled using current techniques, able to meet or beat spacing and concentricities of current molybdenum designs, able to survive NSTAR Acceptance Test vibration levels, and is definitively more resistant to erosion than current molybdenum grids.

\section{Acknowledgments}

The authors would like to thank Dr. J. Steven Snyder and Dr. Dan Goebel of JPL for their guidance in vibration testing and voltage holdoff testing. The authors would also like to thank Dr. John Williams and his team of graduate students at Colorado State University for their work on sputter testing. Many thanks to Larry Martin of Boeing EDD for assembling and recording detailed data, and Bill Quon, also of Boeing EDD, for initial modeling of the PG grids. Lastly, the authors would like to gratefully acknowledge Randy Baggett of NASA MSFC for his support and guidance on the CBIO Program. 


\section{References}

'T. Haag and G. Soulas, "Performance of $8 \mathrm{~cm}$ Pyrolytic Graphite Ion Thruster Optics", AIAA-2002-4335, 38 ${ }^{\text {th }}$ Joint Propulsion Conference, Indianapolis, IN, 2002.

${ }^{2}$ T. Haag and G. Soulas, "Performance and Vibration of 30-cm Pyrolytic Ion Thruster Optics", AIAA-2003-4557, 39 Joint Propulsion Conference, Huntsville, AL, 2003.

${ }^{3}$ J.S. Snyder, J.R. Brophy, D.M. Goebel, J.S. Beatty, and M.K. De Pano, "Development and Testing of Carbon-Based Ion Optics for 30-cm Ion Thrusters", AIAA-2003-4716, 39 Joint Propulsion Conference, Huntsville, AL, 2003.

${ }^{4} J . S$. Snyder and J.R. Brophy, "Performance Characterization and Vibration Testing of 30-cm Carbon-Carbon Ion Optics", AIAA-2004-3959, 40 ${ }^{\text {th }}$ Joint Propulsion Conference, Fort Lauderdale, FL, 2004.

${ }^{5}$ D.M. Laufer, J.D. Williams, C.C. Famell, P.B. Shoemaker, and P.J. Wilbur, "Experimental Evaluation of Sub-Scale CBIO Ion Optics Systems," AIAA-2003-5165, 39 ${ }^{\text {th }}$ Joint Propulsion Conference, Huntsville, AL, 2003.

${ }^{6}$ H.O. Pierson, Handbook of Carbon, Graphite, Diamond and Fullerenes, Noyes Publications, New York, 1993, Chaps. 3, 4.

7J.A. Christensen, et al., "Design and Fabrication of a Flight Model $2.3 \mathrm{~kW}$ Ion Thruster for the Deep Space 1 Mission", AIAA-98-3327, July, 1998.

${ }^{8}$ J.D. Williams, M.L. Johnson, and D.D. Williams, "Differential Sputtering Behavior of Various Forms of Carbon Under Xenon Bombardment," AIAA-2004-3788, 40 ${ }^{\text {th }}$ Joint Propulsion Conference, Fort Lauderdale, FL, 2004. 